\title{
Probiotics: Reiterating What They Are and What They Are Not
}

\author{
Gregor Reid ${ }^{1,2 *}$, Azza A. Gadir ${ }^{3,4}$ and Raja Dhir ${ }^{5}$ \\ ${ }^{1}$ Canadian R\&D Centre for Human Microbiome and Probiotics, Lawson Health Research Institute, London, ON, Canada, \\ ${ }^{2}$ Departments of Microbiology and Immunology and Surgery, Western University, London, ON, Canada, ${ }^{3}$ Division \\ of Immunology, Boston Children's Hospital, Boston, MA, United States, ${ }^{4}$ Department of Pediatrics, Harvard Medical School, \\ Boston, MA, United States, ${ }^{5}$ Seed, Los Angeles, CA, United States
}

\section{OPEN ACCESS}

Edited by:

Paloma López,

Centro de Investigaciones Biológicas

(CIB), Spain

Reviewed by:

Miguel Gueimonde,

Institute of Dairy Products of Asturias (IPLA), Spain

Giuseppe Spano,

University of Foggia, Italy Konstantinos Papadimitriou, Agricultural University of Athens, Greece

${ }^{*}$ Correspondence:

Gregor Reid gregor@uwo.ca

Specialty section:

This article was submitted to

Food Microbiology,

a section of the journal

Frontiers in Microbiology

Received: 22 December 2018

Accepted: 18 February 2019

Published: 12 March 2019

Citation:

Reid G, Gadir AA and Dhir R (2019) Probiotics: Reiterating What

They Are and What They Are Not.

Front. Microbiol. 10:424.

doi: 10.3389/fmicb.2019.00424
It has been over seventeen years since the scientific definition of probiotics was crafted, along with guidelines ensuring the appropriate use of the term. This definition is now used globally, yet on a consistent basis scientists, media and industry misrepresent probiotics or make generalized statements that illustrate a misunderstanding of their utility and limitations. The rate of discovery of novel organisms with potentially therapeutic benefit for both human and environmental health is progressing at an unprecedented rate. However, the term "probiotic" is often misapplied to describe any microbe with plausible therapeutic utility in the human host. It is argued that strict compliance to the scientific definition of the term "probiotic" and avoidance of generalizations to the whole field of probiotics based upon studies of one product, will help advance the development and validation of microbial therapies, and applications to improve human health.

Keywords: human microbiome, probiotics, scientific definition, stewardship, requirements, human health, microbial therapies, translational microbiology

\section{INTRODUCTION}

In 2001, an Expert Panel was convened at the request of the Food and Agriculture Organization of the United Nations, and supported by the World Health Organization, to clarify the definition of probiotics. The resultant publication defined probiotics as "Live microorganisms which when administered in adequate amounts confer a health benefit on the host" (FAO/WHO, 2002). In 2014, a consensus panel reiterated the definition, with one small change replacing "which" with "that" (Hill et al., 2014).

Since 2001, scientific investigation of probiotics has grown substantially. As of February 11, 2019, there were 20,315 papers indexed to the term "probiotic" compared to 760 papers prior to 2001. Commercially, the sales of probiotics are over $\$ 40$ billion and are projected to reach over $\$ 64$ billion by 2023 (Global Market Insights, 2016).

The probiotic field represents the translational potential of microbiology to humans and animals. Despite well characterized mechanisms for microbial therapies to affect a number of organ systems in the host, confusion persists around the precise conditions necessary for a single microbe or microbial consortia to claim probiotic effect (verb) or qualify as a probiotic (noun). Specifically, a set of guidelines stipulates the need for both microbial strain designation and for at least one human study to be performed on the target host to be considered probiotic (FAO/WHO, 2002). Liberal and loose usage of the term has resulted in confusion and as a result, probiotics have collectively garnered a degree of global skepticism. The aim of this article is to clarify the term 
probiotics and urge researchers to employ precision and consistency in both their investigations and communication of data pertaining to microbes for human health.

\section{ILLUSTRATING THE PROBLEMS}

A physician wanting to provide a patient with advice on preventing traveler's diarrhea (TD) may have referenced a recent meta-analysis by Bae (2018) regarding the use of probiotics as a treatment. This publication used a seemingly appropriate inclusion criteria of double-blind, placebocontrolled, randomized human trials with TD as an outcome, and various statistical methods to conclude that probiotics were efficacious in preventing TD. However, a closer look at the listed interventions shows one study that utilized dead lactobacilli and two other studies that included the administration of prebiotics. Caution must be exercised when interpreting such data, given that probiotics must be live (FAO/WHO, 2002; Sanders et al., 2007), and the definition of a prebiotic is "a substrate that is selectively utilized by host microorganisms conferring a health benefit" (Gibson et al., 2017).

Confusion within the field is not restricted to meta-analyses. Conclusions are often advanced based on improperly designed intervention studies that extrapolate broad generalizations from a limited data set. Several examples are worth citing. One study concentrating on the use of probiotics in acute pancreatitis suggested that they contributed to bacterial translocation and enterocyte damage in organ failure (Besselink et al., 2004, 2009). Yet, there is no direct evidence that the administered strains were the cause (Reid et al., 2008). Aside from the issue of whether or not this is an appropriate clinical setting to administer extrinsic living bacteria, the study's conclusion has been enlarged to imply that any or all probiotics might adversely impact all patients with pancreatitis. Nine years later, that initial study has been fully reassessed concluding that probiotics are not contraindicated in pancreatitis. The prior negative outcomes were related to unexpected lactic acidosis from the fermentation of carbohydrates (Bongaerts and Severijnen, 2016). Indeed, this more recent study recommended starting probiotics immediately upon diagnosis, massively increasing the probiotic dose, and limiting fermentable carbohydrates.

To avoid unwarranted damage to the reputation of probiotics, stringent testing and careful clinical guidelines are imperative in the treatment and management of high risk adults and children (Sanders et al., 2016). Not all centers have avoided use of probiotics in seriously ill patients as a result of the pancreatitis paper, and indeed one site has reported substantial benefits in treating seriously ill trauma patients with probiotics or synbiotics (Giamarellos-Bourboulis et al., 2009). Furthermore, there is clear evidence of probiotics as useful in preventing necrotizing enterocolitis in premature, low-birth weight babies which is certainly a critically ill patient sub-set (Patel and Underwood, 2018).

Another pertinent example of the over interpretation of limited data is a recent study conducted on 34 patients suffering from "brain fogginess," which is a set of presentations including impaired concentration, confusion, and poor short-term memory (Rao et al., 2018). Based on the observation that consumption of fermented food or commercially available microbial products was a shared trait amongst the patients reporting brain fog, the study implied that all probiotics could be causative agents of brain fogginess. While the definition of probiotics accommodates strains of any taxa, it is unlikely that these included the Streptococcus, Staphylococcus, Neisseria, or Hemophilus, which were the primary organisms detected by culture in the duodenal aspirates. Furthermore, $68 \%$ of the cohort presented with small intestinal bacterial overgrowth (SIBO) and there were no data to verify patients were acidotic; a crucial linkage considering many lactobacilli and all bifidobacteria only produce L-lactate and not D-lactate (Vitetta et al., 2017; Petrova et al., 2018; Quigley et al., 2018). In the future, appropriate trial design can strengthen the field by moving away from observational and correlative data sets. In this case, the study concluded that probiotics may be causative of brain fog on the observation that antibiotic therapy alleviated symptoms of brain fog, whereas a more appropriately designed study would have split the patients after antibiotic therapy into two groups, and upon reintroduction of probiotics in a defined cohort, compared the emergence of symptoms against the antibiotic-treated control.

In a third example, a recent study suggesting probiotics impair naïve microbiota recovery after antibiotic therapy concluded that probiotics may be harmful to the gut microbiota when administered after antibiotic use (Suez et al., 2018). In this instance, Suez et al. (2018) performed an intervention study on healthy subjects given high doses of metronidazole and ciprofloxacin. The subjects received evacuation therapy twice in 28 days so that intestinal samples could be acquired. Based on the observation that 10-15 species had a longer recovery time compared to spontaneous recovery and autologous fecal microbiota transplantation (aFMT), the study put forward the thesis that probiotic consumption may present latent danger to the gut microbiome and, by extension, the host. There are serious limitations to asserting broad generalizations from one narrow data set to the field as a whole. In this case, two subsequent human studies published within a month of the Suez et al. (2018) study and a second report from that group (Zmora et al., 2018) presented alternative data (De Wolfe et al., 2018; Korpela et al., 2018). The first demonstrated that probiotic supplementation restored normal microbiota composition and function in antibiotic-treated and cesarean-born infants. A second study examined a different microbial preparation and its effect on microbiome recovery after antibiotic therapy (De Wolfe et al., 2018). Notably, the microbial intervention contained a $700 \%$ higher dosage and was administered for twice the intervention period as the Suez et al. (2018) intervention with opposite data showing improved microbiome alpha-diversity in the "probiotics" group as measured by the Shannon diversity index, a heterogeneity measure that combines richness and evenness components of microbial diversity (De Wolfe et al., 2018). It is worth noting that all three publications were released in the same month, yet the results of one were extrapolated to the field as a whole, hindering an open scientific dialog on the advantages or 
limitations of probiotics or their reproducible effects based on strain specificity.

Relevantly, it is worth nothing that one should not rely on metagenomic and 16s rRNA data as an endpoint alone, but rather monitor clinical and functional outcomes, for example an increase in short-chain fatty acid production and their direct immunological effect on colonic Treg cells (Smith et al., 2013) and other pathological processes (Cox et al., 2009; Ho et al., 2018; Nagpal et al., 2018). Another effect of well characterized probiotic strains is to confirm protection of the intestinal epithelium, in some cases reversing damage caused by antibiotics (Resta-Lenert and Barrett, 2006).

\section{THE MINIMAL REQUIREMENTS FOR THE EVALUATION OF AN EFFECTIVE PROBIOTIC}

As indicated in the original FAO/WHO (2002) report, there are certain expectations required to call an organism "probiotic." These have been further clarified in 2014 (Hill et al., 2014), and clearly must include:

The definition of probiotics as "Live microorganisms, that when administered in adequate amounts, confer a health benefit on the host."

1. That microbes must be alive in an adequate number when administered.

2. Strains must be identified genetically, classified using the latest terminology, and designated by numbers, letters, or names.

3. Appropriately sized and designed studies must be performed to designate a strain as probiotic and using the strain(s) on the host to which the probiotics are intended (human, livestock, companion animal, etc).
4. Strains shown to confer a benefit for one condition may not be probiotic for another application.

5. Strains that are probiotic for humans but are being used in animal studies should be clearly designated as human probiotics under experimental testing.

Fermented foods, prebiotics, fecal microbiota transplant, and microbial strains of the same genus or species as documented probiotic strains but have not undergone appropriate testing on the target host should not be considered as probiotics based on adherence to the scientific definition.

\section{GUIDANCE FOR PHYSICIANS}

Within the field of microbiome science, the rate of discovery of novel organisms with potentially therapeutic benefit for the human host is progressing rapidly. Emerging research on microbial strains includes some with systemic immunomodulatory activity (Geva-Zatorsky et al., 2017), the prevention and treatment of food allergies (Kim et al., 2017; Feehley et al., 2019), modulation of the gut-liver axis (Bajaj, 2019), production of neuroactive metabolites (Valles-Colomer et al., 2019), and inhibitory activity against infectious microbes in the gut (Kau et al., 2011), skin (Kober and Bowe, 2015), and urogenital tract (Gottschick et al., 2017). Furthermore, microbes are now understood as integral to an extensive number of essential metabolic processes (Table 1) (Hentzer and Givskov, 2003; Ley et al., 2006; Abubucker et al., 2012; Karlsson et al., 2012; Morgan et al., 2012; den Besten et al., 2013; Tang et al., 2013; Tang and Hazen, 2014; Fiorucci and Distrutti, 2015; Hartstra et al., 2015; Magnúsdóttir et al., 2015; McCabe et al., 2015; Neuman et al., 2015; O’Mahony et al., 2015; Yano et al., 2015; Savidge, 2016; Sonnenburg and Bäckhed, 2016; Thaiss et al., 2016; Yan et al., 2016; Zeng et al., 2016; Blander et al., 2017; Koppel et al., 2017; Weiss and Hennet, 2017; Desselberger, 2018; Fukui et al., 2018;

TABLE 1 | Physiological and metabolic processes influenced by the human microbiome.

\begin{tabular}{|c|c|}
\hline Processes & Reference \\
\hline Enzymatic pathways, glycosaminoglycan degradation & Abubucker et al., 2012; Koppel et al., 2017 \\
\hline Energy metabolism (short chain fatty acids, glucose) & $\begin{array}{l}\text { den Besten et al., 2013; Hartstra et al., 2015; Sonnenburg and } \\
\text { Bäckhed, } 2016\end{array}$ \\
\hline Neurotransmitter production & O’Mahony et al., 2015; Yano et al., 2015; Savidge, 2016 \\
\hline Vitamin absorption & Magnúsdóttir et al., 2015 \\
\hline $\begin{array}{l}\text { Regulation of bile acid metabolism, (deoxycholic and lithocholic acids, } \\
\text { bile salts) }\end{array}$ & Fiorucci and Distrutti, 2015; Weiss and Hennet, 2017 \\
\hline Endocrine and gut hormone regulation & Neuman et al., 2015; Fukui et al., 2018 \\
\hline Adaptive immunity, mucosal and systemic immunity & Thaiss et al., 2016; Desselberger, 2018 \\
\hline Cell proliferation, mucosal barrier protection, inflammation & Morgan et al., 2012; Blander et al., 2017; Schirmer et al., 2018 \\
\hline Protection against pathogens & Zeng et al., 2016; Zhang et al., 2018 \\
\hline $\begin{array}{l}\text { Vascularization, tri-ethylamine associated atherosclerosis, tri-ethylamine } \\
\mathrm{N} \text {-oxide (TMAO) production }\end{array}$ & Karlsson et al., 2012; Tang et al., 2013; Tang and Hazen, 2014 \\
\hline Bone mass & McCabe et al., 2015; Yan et al., 2016 \\
\hline Appetite signaling, obesity & Hentzer and Givskov, 2003; Ley et al., 2006 \\
\hline $\begin{array}{l}\text { Metabolic transformation of xenobiotics (small molecules foreign to the } \\
\text { body) }\end{array}$ & Koppel et al., 2017 \\
\hline
\end{tabular}


Schirmer et al., 2018; Zhang et al., 2018). From these discoveries will emerge new microbial-based interventions.

Therefore, it is expected that the misuse of the term "probiotic" to describe any microbe with plausible therapeutic utility in the human host may persist unless there is strict adherence to the requirements of an exacting definition. To the extent that physicians may require guidance for evaluating the merits of current and future microbial preparations, three key elements in determining the validity of a probiotic are proposed:

1. Evidence that the strain(s) has been tested in a randomized, controlled or equivalent human trial, in either a heterogeneous population or stratified based on defined characteristics of host or microbial genomics.

2. The dose and viability in the product are equal to that of the human trial(s).

3. Whole genome strain characterization and transparently declared strain designation are provided.

If a strain is selected for a specific mechanism, such as being able to upregulate gut barrier proteins, and is then tested in humans and shown to improve gut barrier function leading to a health benefit (Iemoli et al., 2012), then inclusion of that strain at the tested dose would be expected to convey the same outcome even in the presence of other strains. An example is B. breve BR03 being selected for immunomodulatory and gut barrier function activities and consequently being found in a 300-person study to increase Treg cells, or in 49 children with Celiac disease to decrease TNF $\alpha$ production (Del Piano et al., 2010; Klemenak et al., 2015).

Studies of the human microbiome have established that despite significant interpersonal variation at the species level, many core metabolic functions are maintained amongst individuals in a population (Bogiatzi et al., 2018; Kleerebezem et al., 2018; Wandro et al., 2018). Therefore, as the field of personalized medicine gains traction, it is important that proponents of individualized therapies clearly define the basis for population stratification, and demonstrate efficacy for the proposed personalized therapy in the specific subpopulation being targeted. A more universal approach would entail the discovery and validation of microbes with significant and reproducible effects across a heterogeneous population.

\section{REFERENCES}

Abubucker, S., Segata, N., Goll, J., Schubert, A. M., Izard, J., Cantarel, B. L., et al. (2012). Metabolic reconstruction for metagenomic data and its application to the human microbiome. PLoS Comp. Biol. 8:e1002358. doi: 10.1371/journal. pcbi. 1002358

Bae, J. M. (2018). Prophylactic efficacy of probiotics on travelers' diarrhea: an adaptive meta-analysis of randomized controlled trials. Epidemiol. Health 40:e2018043. doi: 10.4178/epih.e2018043

Bajaj, J. S. (2019). Alcohol, liver disease and the gut microbiota. Nat. Rev. Gastroenterol. Hepatol. doi: 10.1038/s41575-018-0099-1 [Epub ahead of print].

Besselink, M. G., Timmerman, H. M., Buskens, E., Nieuwenhuijs, V. B., Akkermans, L. M., Gooszen, H. G., et al. (2004). Probiotic prophylaxis in patients with predicted severe acute pancreatitis (PROPATRIA): design and rationale of a double-blind, placebo-controlled randomised
Therapies under both a stratified and heterogeneous population would meet the guidelines of probiotic, provided the impact on human health is established in a controlled human trial.

\section{FUTURE DIRECTIONS}

Research investigating microbial administration to, or modulation of, the human microbiome to improve health has been progressing at a rapid rate since the term probiotics was defined in 2001. It has been amplified by the Human Microbiome Project and sustained by the interdisciplinary efforts beyond microbiology that have contributed to the velocity of microbiome research worldwide. It comes at a time where there has never been a greater appreciation for the microbes that inhabit life-forms and the planet. The potential to manipulate these microbial ecosystems offers great hope for new preventive and treatment options for many diseases. The idea that ingesting or administering live microbes can serve therapeutic functions is gaining prominence, but just as too many microbiome studies in rodents and humans "oversell" their limited findings (Hooks et al., 2018), too much misinformation has stemmed from misuse of the probiotic term. It is hoped that the fundamental principles outlined herein, that encourage adherence to a strict scientific definition of "probiotics" and rigorous assessment of their actual clinical outcomes will assert greater clarity and foster a deeper understanding within this dynamic field.

\section{AUTHOR CONTRIBUTIONS}

GR and $\mathrm{RD}$ conceived and wrote the original version of the manuscript. GR, RD, and AG revised the manuscript to meet the reviewers' comments.

\section{ACKNOWLEDGMENTS}

The authors would like to acknowledge the other members of the United Nations - World Health Organizational Expert Panel that defined the term 'probiotics' in 2001.

multicenter trial [ISRCTN38327949]. BMC Surg. 4:12. doi: 10.1186/14712482-4-12

Besselink, M. G., van Santvoort, H. C., Renooij, W., de Smet, M. B., Boermeester, M. A., Fischer, K., et al. (2009). Intestinal barrier dysfunction in a randomized trial of a specific probiotic composition in acute pancreatitis. Ann. Surg. 250, 712-719. doi: 10.1097/SLA.0b013e3181bce5bd

Blander, J. M., Longman, R. S., Iliev, I. D., Sonnenberg, G. F., and Artis, D. (2017). Regulation of inflammation by microbiota interactions with the host. Nat. Immunol. 18:851. doi: 10.1038/ni.3780

Bogiatzi, C., Gloor, G., Allen-Vercoe, E., Reid, G., Wong, R. G., Urquhart, B. L., et al. (2018). Metabolic products of the intestinal microbiome and extremes of atherosclerosis. Atherosclerosis 273, 91-97. doi: 10.1016/j.atherosclerosis.2018. 04.015

Bongaerts, G. P., and Severijnen, R. S. (2016). A reassessment of the PROPATRIA study and its implications for probiotic therapy. Nat. Biotechnol. 34, 55-63. doi: $10.1038 /$ nbt. 3436 
Cox, M. A., Jackson, J., Stanton, M., Rojas-Triana, A., Bober, L., Laverty, M., et al. (2009). Short-chain fatty acids act as anti-inflammatory mediators by regulating prostaglandin E (2) and cytokines. World J. Gastroenterol. 15, 5549-5557. doi: 10.3748/wjg.15.5549

De Wolfe, T. J., Eggers, S., Barker, A. K., Kates, A. E., Dill-McFarland, K. A., Suen, G., et al. (2018). Oral probiotic combination of Lactobacillus and Bifidobacterium alters the gastrointestinal microbiota during antibiotic treatment for Clostridium difficile infection. PLoS One 13:e0204253. doi: 10. 1371/journal.pone.0204253

Del Piano, M., Carmagnola, S., Anderloni, A., Andorno, S., Ballarè, M., Balzarini, M., et al. (2010). The use of probiotics in healthy volunteers with evacuation disorders and hard stools: a double-blind, randomized, placebocontrolled study. J. Clin. Gastroenterol. 44(Suppl. 1), S30-S34. doi: 10.1097/ MCG.0b013e3181ee31c3

den Besten, G., van Eunen, K., Groen, A. K., Venema, K., Reijngoud, D. J., and Bakker, B. M. (2013). The role of short-chain fatty acids in the interplay between diet, gut microbiota and host energy metabolism. J. Lipid Res. 54, 2325-2340. doi: $10.1194 /$ jlr.R036012

Desselberger, U. (2018). The mammalian intestinal microbiome: composition, interaction with the immune system, significance for vaccine efficacy, and potential for disease therapy. Pathogens 7:E57. doi: 10.3390/pathogens 7030057

FAO/WHO (2002). Guidelines for the Evaluation of Probiotics in Food. Available At: https://www.who.int/foodsafety/fs_management/en/probiotic_guidelines.pdf

Feehley, T., Plunkett, C. H., Bao, R., Choi Hong, S. M., Culleen, E., BeldaFerre, P., et al. (2019). Healthy infants harbor intestinal bacteria that protect against food allergy. Nat. Med. doi: 10.1038/s41591-018-0324-Z [Epub ahead of print].

Fiorucci, S., and Distrutti, E. (2015). Bile acid-activated receptors, intestinal microbiota, and the treatment of metabolic disorders. Trends Mol. Med. 21, 702-714. doi: 10.1016/j.molmed.2015.09.001

Fukui, H., Xu, X., and Miwa, H. (2018). Role of gut microbiota-gut hormone axis in the pathophysiology of functional gastrointestinal disorders. J. Neurogastroenterol. Motil. 24, 367-386. doi: 10.5056/jnm18071

Geva-Zatorsky, N., Sefik, E., Kua, L., Pasman, L., Tan, T. G., Ortiz-Lopez, A., et al. (2017). Mining the human gut microbiota for immunomodulatory organisms. Cell 168, 928-943. doi: 10.1016/j.cell.2017.01.022

Giamarellos-Bourboulis, E. J., Bengmark, S., Kanellakopoulou, K., and Kotzampassi, K. (2009). Pro- and synbiotics to control inflammation and infection in patients with multiple injuries. J. Trauma 67, 815-821. doi: 10.1097/TA.0b013e31819d979e

Gibson, G. R., Hutkins, R., Sanders, M. E., Prescott, S., Reimer, R., Salminen, S., et al. (2017). Expert consensus document: the International Scientific Association for Probiotics and Prebiotics (ISAPP) consensus statement on the term and scope of prebiotics. Nat. Rev. Gastroenterol. Hepatol. 14, 1-12. doi: 10.1038/nrgastro.2017.75

Global Market Insights (2016). Probiotics Market Size to Exceed USD 64 Billion by 2023: Global Market Insights Inc. Available at: https: //www.prnewswire.com/news-releases/probiotics-market-size-to-exceedusd-64-billion-by-2023-global-market-insights-inc-578769201.html

Gottschick, C., Deng, Z., Vital, M., Masur, C., Abels, C., Pieper, D. H., et al. (2017). The urinary microbiota of men and women and its changes in women during bacterial vaginosis and antibiotic treatment. Microbiome 5:99. doi: 10.1186/ s40168-017-0305-3

Hartstra, A. V., Bouter, K. E., Bäckhed, F., and Nieuwdorp, M. (2015). Insights into the role of the microbiome in obesity and type 2 diabetes. Diabetes Care 38, 159-165. doi: $10.2337 / \mathrm{dc} 14-0769$

Hentzer, M., and Givskov, M. (2003). Pharmacological inhibition of quorum sensing for the treatment of chronic bacterial infections. J. Clin. Invest. 112, 1300-1307. doi: 10.1172/JCI20074

Hill, C., Guarner, F., Reid, G., Gibson, G. R., Merenstein, D., Pot, B., et al. (2014). The International Scientific Association for Probiotics and Prebiotics consensus statement on the scope and appropriate use of the term probiotics. Nat. Rev. Gastroenterol. Hepatol. 11, 506-514. doi: 10.1038/nrgastro.2014.66

Ho, L., Ono, K., Tsuji, M., Mazzola, P., Singh, R., and Pasinetti, G. M. (2018). Protective roles of intestinal microbiota derived short chain fatty acids in Alzheimer's disease-type beta-amyloid neuropathological mechanisms. Expert Rev. Neurother. 18, 83-90. doi: 10.1080/14737175.2018.1400909
Hooks, K. B., Konsman, J. P., and O’Malley, M. A. (2018). Microbiota-gutbrain research: a critical analysis. Behav. Brain Sci. [Epub ahead of print]. doi: 10.1017/S0140525X18002133

Iemoli, E., Trabattoni, D., Parisotto, S., Borgonovo, L., Toscano, M., Rizzardini, G., et al. (2012). Probiotics reduce gut microbial translocation and improve adult atopic dermatitis. J. Clin. Gastroenterol. 46(Suppl.), S33-S40. doi: 10.1097/ MCG.0b013e31826a8468

Karlsson, F. H., Fåk, F., Nookaew, I., Tremaroli, V., Fagerberg, B., Petranovic, D., et al. (2012). Symptomatic atherosclerosis is associated with an altered gut metagenome. Nat. Commun. 3:1245. doi: 10.1038/ncomms2266

Kau, A. L., Ahern, P. P., Griffin, N. W., Goodman, A. L., and Gordon, J. I. (2011). Human nutrition, the gut microbiome and the immune system. Nature 474, 327-336. doi: 10.1038/nature10213

Kim, Y. G., Sakamoto, K., Seo, S. U., Pickard, J. M., Gillilland, M. G. III, Pudlo, N. A., et al. (2017). Neonatal acquisition of Clostridia species protects against colonization by bacterial pathogens. Science 356, 315-319. doi: 10.1126/science. aag2029

Kleerebezem, M., Binda, S., Bron, P. A., Gross, G., Hill, C., van Hylckama Vlieg, J. E., et al. (2018). Understanding mode of action can drive the translational pipeline towards more reliable health benefits for probiotics. Curr. Opin. Biotechnol. 56, 55-60. doi: 10.1016/j.copbio.2018.09.007

Klemenak, M., Dolinšek, J., Langerholc, T., Di Gioia, D., and Mičetić-Turk, D. (2015). Administration of Bifidobacterium breve decreases the production of TNF- $\alpha$ in children with Celiac Disease. Dig. Dis. Sci. 60, 3386-3392. doi: 10.1007/s10620-015-3769-7

Kober, M., and Bowe, W. P. (2015). The effect of probiotics on immune regulation, acne, and photoaging. Int. J. Womens Dermatol. 1, 85-89. doi: 10.1016/j.ijwd. 2015.02.001

Koppel, N., Rekdal, V. M., and Balskus, E. P. (2017). Chemical transformation of xenobiotics by the human gut microbiota. Science 356:eaag2770. doi: 10.1126/ science.aag 2770

Korpela, K., Salonen, A., Vepsäläinen, O., Suomalainen, M., Kolmeder, C., Varjosalo, M., et al. (2018). Probiotic supplementation restores normal microbiota composition and function in antibiotic-treated and in caesareanborn infants. Microbiome 6:182. doi: 10.1186/s40168-018-0567-4

Ley, R. E., Turnbaugh, P. J., Klein, S., and Gordon, J. I. (2006). Microbial ecology: human gut microbes associated with obesity. Nature 444, 1022-1023. doi: 10.1038/4441022a

Magnúsdóttir, S., Ravcheev, D., de Crécy-Lagard, V., and Thiele, I. (2015). Systematic genome assessment of B-vitamin biosynthesis suggests co-operation among gut microbes. Front. Genet. 6:148. doi: 10.3389/fgene.2015.00148

McCabe, L., Britton, R. A., and Parameswaran, N. (2015). Prebiotic and probiotic regulation of bone health: role of the intestine and its microbiome. Curr. Osteoporos. Rep. 13, 363-371. doi: 10.1007/s11914-015-0292-x

Morgan, X. C., Tickle, T. L., Sokol, H., Gevers, D., Devaney, K. L., and Ward, D. V. (2012). Dysfunction of the intestinal microbiome in inflammatory bowel disease and treatment. Genome Biol. 13:R79. doi: 10.1186/gb-201213-9-r79

Nagpal, R., Wang, S., Ahmadi, S., Hayes, J., Gagliano, J., Subashchandrabose, S., et al. (2018). Human-origin probiotic cocktail increases short-chain fatty acid production via modulation of mice and human gut microbiome. Sci Rep. 8:12649. doi: 10.1038/s41598-018-30114-4

Neuman, H., Debelius, J. W., Knight, R., and Koren, O. (2015). Microbial endocrinology: the interplay between the microbiota and the endocrine system. FEMS Microbiol. Rev. 39, 509-521. doi: 10.1093/femsre/fuu010

O’Mahony, S. M., Clarke, G., Borre, Y. E., Dinan, T. G., and Cryan, J. F. (2015). Serotonin, tryptophan metabolism and the brain-gut-microbiome axis. Behav. Brain Res. 277, 32-48. doi: 10.1016/j.bbr.2014.07.027

Patel, R. M., and Underwood, M. A. (2018). Probiotics and necrotizing enterocolitis. Semin. Pediatr. Surg. 27, 39-46. doi: 10.1053/j.sempedsurg.2017. 11.008

Petrova, M. I., Macklaim, J., Wuyts, S., Verhoeven, T., Vanderleyden, J., Gloor, G. B., et al. (2018). Comparative genomic and phenotypic analysis of the vaginal probiotic Lactobacillus rhamnosus GR-1. Front. Microbiol. 9:1278. doi: 10.3389/ fmicb.2018.01278

Quigley, E. M. M., Pot, B., and Sanders, M. E. (2018). 'Brain fogginess' and D-lactic acidosis: probiotics are not the cause. Clin. Transl. Gastroenterol. 9:187. doi: 10.1038/s41424-018-0057-9 
Rao, S. S. C., Rehman, A., Yu, S., and Andino, N. M. (2018). Brain fogginess, gas and bloating: a link between SIBO, probiotics and metabolic acidosis. Clin. Transl. Gastroenterol. 9:162. doi: 10.1038/s41424-018-0030-7

Reid, G., Gibson, G., Sanders, M. E., Guarner, F., and Versalovic, J. (2008). Probiotic prophylaxis in predicted severe acute pancreatitis. Lancet 372, 112 113. doi: 10.1016/S0140-6736(08)61024-7

Resta-Lenert, S., and Barrett, K. E. (2006). Probiotics and commensals reverse TNF-alpha- and IFN-gamma-induced dysfunction in human intestinal epithelial cells. Gastroenterology 130, 731-746. doi: 10.1053/j.gastro.2005. 12.015

Sanders, M. E., Hamilton, J., Reid, G., and Gibson, G. (2007). A nonviable preparation of Lactobacillus acidophilus is not a probiotic. Clin. Infect. Dis. 44:886.

Sanders, M. E., Merenstein, D. J., Ouwehand, A. C., Reid, G., Salminen, S., Cabana, M. D., et al. (2016). Probiotic use in at-risk populations. J. Am. Pharm. Assoc. 56, 680-686. doi: 10.1016/j.japh.2016.07.001

Savidge, T. C. (2016). Epigenetic regulation of enteric neurotransmission by gut bacteria. Front. Cell. Neurosci. 9:503. doi: 10.3389/fncel.2015.00503

Schirmer, M., Franzosa, E. A., Lloyd-Price, J., McIver, L. J., Schwager, R., and Poon, T. W. (2018). Dynamics of metatranscription in the inflammatory bowel disease gut microbiome. Nat. Microbiol. 3, 337-346. doi: 10.1038/s41564017-0089-z

Smith, P. M., Howitt, M. R., Panikov, N., Michaud, M., Gallini, C. A., BohloolyY, M., et al. (2013). The microbial metabolites, short-chain fatty acids, regulate colonic Treg cell homeostasis. Science 341, 569-573. doi: 10.1126/science. 1241165

Sonnenburg, J. L., and Bäckhed, F. (2016). Diet-microbiota interactions as moderators of human metabolism. Nature 535, 56-64. doi: 10.1038/ nature 18846

Suez, J., Zmora, N., Zilberman-Schapira, G., Mor, U., Dori-Bachash, M., Bashiardes, S., et al. (2018). Post-antibiotic gut mucosal microbiome reconstitution is impaired by probiotics and improved by autologous FMT. Cell 174, 1406-1423.e16. doi: 10.1016/j.cell.2018.08.047

Tang, W. H., Wang, Z., Levison, B. S., Koeth, R. A., Britt, E. B., Fu, X., et al. (2013). Intestinal microbial metabolism of phosphatidylcholine and cardiovascular risk. N. Engl. J. Med. 368, 1575-1584. doi: 10.1056/NEJMoa1109400

Tang, W. W., and Hazen, S. L. (2014). The contributory role of gut microbiota in cardiovascular disease. J. Clin. Invest. 124, 4204-4211. doi: 10.1172/JCI7 2331

Thaiss, C. A., Zmora, N., Levy, M., and Elinav, E. (2016). The microbiome and innate immunity. Nature 535, 65-74. doi: 10.1038/nature18847

Valles-Colomer, M., Falony, G., Darzi, Y., Tigchelaar, E. F., Wang, J., Tito, R. Y., et al. (2019). The neuroactive potential of the human gut microbiota in quality of life and depression. Nat. Microbiol. doi: 10.1038/s41564-018-0337-x [Epub ahead of print].

Vitetta, L., Coulson, S., Thomsen, M., Nguyen, T., and Hall, S. (2017). Probiotics, D-Lactic acidosis, oxidative stress and strain specificity. Gut Microbes 8, 311322. doi: 10.1080/19490976.2017.1279379

Wandro, S., Osborne, S., Enriquez, C., Bixby, C., Arrieta, A., and Whiteson, K. (2018). The microbiome and metabolome of preterm infant stool are personalized and not driven by health outcomes, including necrotizing enterocolitis and late-onset sepsis. mSphere 3, e104-18. doi: 10.1128/mSphere. 00104- 18

Weiss, G. A., and Hennet, T. (2017). Mechanisms and consequences of intestinal dysbiosis. Cell. Mol. Life Sci. 74, 2959-2977. doi: 10.1007/s00018-017-2509-x

Yan, J., Herzog, J. W., Tsang, K., Brennan, C. A., Bower, M. A., and Garrett, W. S. (2016). Gut microbiota induce IGF-1 and promote bone formation and growth. Proc. Nat. Acad. Sci. U.S.A. 113, E7554-E7563. doi: 10.1073/pnas.1607235113

Yano, J. M., Yu, K., Donaldson, G. P., Shastri, G. G., Ann, P., and Ma, L. (2015). Indigenous bacteria from the gut microbiota regulate host serotonin biosynthesis. Cell 161, 264-276. doi: 10.1016/j.cell.2015.02.047

Zeng, M. Y., Cisalpino, D., Varadarajan, S., Hellman, J., Warren, H. S., and Cascalho, M. (2016). Gut microbiota-induced immunoglobulin G controls systemic infection by symbiotic bacteria and pathogens. Immunity 44, 647-658. doi: 10.1016/j.immuni.2016.02.006

Zhang, Y., Brady, A., Jones, C., Song, Y., Darton, T. C., Jones, C., et al. (2018). Compositional and functional differences in the human gut microbiome correlate with clinical outcome following infection with wild-type Salmonella enterica serovar Typhi. mBio 9:e686-18. doi: 10.1128/mBio.00686-18

Zmora, N., Zilberman-Schapira, G., Suez, J., Mor, U., Dori-Bachash, M., Bashiardes, S., et al. (2018). Personalized gut mucosal colonization resistance to empiric probiotics is associated with unique host and microbiome features. Cell 174, 1388-1405.e22. doi: 10.1016/j.cell.2018.08.041

Conflict of Interest Statement: RD is a founder in Seed, a biotechnology company developing microbial therapies not discussed in this paper. GR is a scientific advisor to Seed on probiotics. AG is involved in research and development at Seed and developing intellectual property related to microbial regulation of immune mechanisms underlying food allergies, which is not discussed in this paper.

Copyright (C) 2019 Reid, Gadir and Dhir. This is an open-access article distributed under the terms of the Creative Commons Attribution License (CC BY). The use, distribution or reproduction in other forums is permitted, provided the original author(s) and the copyright owner(s) are credited and that the original publication in this journal is cited, in accordance with accepted academic practice. No use, distribution or reproduction is permitted which does not comply with these terms. 